\title{
Screening for alcohol and drug use disorders among adults in primary care: a review
}

\author{
This article was published in the following Dove Press journal: \\ Substance Abuse and Rehabilitation \\ 10 April 2012 \\ Number of times this article has been viewed
}

\author{
Daniel J Pilowsky' \\ Li-Tzy Wu ${ }^{2}$ \\ 'Departments of Epidemiology \\ and Psychiatry, Columbia University, \\ and the New York State Psychiatric \\ Institute, New York City, NY, \\ ${ }^{2}$ Department of Psychiatry and \\ Behavioral Sciences, School of \\ Medicine, Duke University Medical \\ Center, Durham, NC, USA
}

Background: The Patient Protection and Affordable Care Act of 2010 supports integration of substance abuse interventions and treatments into the mainstream health care system. Thus, effective screening and intervention for substance use disorders in health care settings is a priority. Objective: This paper reviews the prevalence of alcohol and drug use disorders (abuse or dependence) in primary care settings and emergency departments, as well as current screening tools and brief interventions.

Methods: MEDLINE was searched using the following keywords: alcohol use, alcohol use disorder, drug use, drug use disorder, screening, primary care, and emergency departments. Using the related-articles link, additional articles were screened for inclusion. This review focuses on alcohol and drug use and related disorders among adults in primary care settings.

Conclusion: Screening, brief intervention, and referral for treatment are feasible and effective in primary care settings, provided that funding for screening is available, along with brief interventions and treatment facilities to which patients can be referred and treated promptly.

Keywords: brief intervention, emergency departments

\section{Introduction}

According to the 2010 National Survey on Drug Use and Health, 70\% of Americans aged 18 years or older reported alcohol use in the past year (an estimated 161 million adults), and $15 \%$ reported illicit or nonmedical drug use in the past year (an estimated 34 million adults). Additionally, 9\% of American adults aged 18 years or older met criteria for an alcohol or drug use disorder (abuse or dependence) in the past year, translating into approximately 20 million adults. ${ }^{1}$ Alcohol and drug use are associated with detrimental physical, social, and psychological consequences. ${ }^{2,3}$

American adults with alcohol and drug use disorders are overrepresented in primary care and emergency department (ED) settings, and therefore these settings are important sites for identifying and briefly intervening with substance abusers. ${ }^{4}$ Alcohol-related problems and alcohol use disorders (AUDs) are common in these settings. ${ }^{5}$ Yet, few adults are asked about alcohol or drug use when they visit a general practitioner. For example, in a survey of 7371 adults reporting a visit to a medical practitioner, slightly over a third of adults aged 18-49 years were asked about alcohol and drug use, and those over age 49 were asked less often. ${ }^{6}$ To address this important health problem, the United States Preventive Services Task Force (USPSTF) issued recommendations for screening and behavioral counseling for alcohol use problems in primary care. ${ }^{7}$ The USPSTF indicates that screening in primary care settings can accurately identify individuals at risk for alcohol-related harmful health consequences, and that brief 
behavioral counseling interventions can lead to small to moderate reductions in alcohol consumption. ${ }^{7}$ However, due to a lack of research on screening, brief intervention, and referral to treatment (SBIRT), routine screening for drug use problems is not recommended. Screening for alcohol use problems in primary care and $\mathrm{EDs}^{8}$ has not been done regularly (eg, in a survey of EDs, only $15 \%$ of department directors reported having formal alcohol-related screening and intervention policies). Perceived barriers to screening by clinicians include limited provider time and limited financial resources. $^{8}$

The Patient Protection and Affordable Care Act of 2010 emphasizes the integration of substance abuse interventions and treatments into the mainstream health care system. Thus, there is hope that its implementation will bring about funding for screening and brief interventions. The objectives of this paper are to (1) provide an update on the prevalence of alcohol and drug use disorders (abuse or dependence; terms used here as defined in the Diagnostic and Statistical Manual of Mental Disorders, Fourth Edition [DSM-IV]) in primary care settings and EDs, (2) review screening tools used in medical settings, and (3) summarize recent findings about screening and brief interventions for alcohol or drug use problems in these settings. Lastly, recent developments in screening and intervention are reviewed.

\section{Prevalence of alcohol and drug use disorders in primary care settings}

Available studies indicate a higher prevalence of alcoholrelated problems and disorders in primary care among men compared with women. ${ }^{5,9}$ A study using the CAGE (a screening instrument described below) reported that $44.8 \%$ of primarycare outpatients tested positive for lifetime alcohol problems, but the prevalence was reduced to $17.2 \%$ when the questions were asked regarding the past year. ${ }^{9}$ Bradley et al define risky drinking as drinking more than 7 and 14 drinks per week for women and men, respectively, or drinking 6 or more drinks on any occasion in the past year. ${ }^{5}$ They define alcohol misuse as meeting criteria for risky drinking and/or meeting criteria for DSM-IV AUDs in the past year. Using these definitions, they found that $19 \%$ and $33 \%$ of women and men, respectively, met criteria for alcohol misuse at an academic family practice clinic in Texas. Past-year DSM-IV AUDs were diagnosed in $9.0 \%$ and $17 \%$ of women and men, respectively, in the same sample of 1319 primary care attendees. ${ }^{5}$ A large survey $(\mathrm{N}=21,282$ adults aged 18-65) of community-based primary care clinic attendees found that 5\% were alcohol dependent and $8 \%$ were considered problem drinkers. While $5 \%$ reported current illicit drug use, 20\% reported using illicit drugs five or more times in their lifetime. ${ }^{10}$ These prevalences are higher than those in the general population. For example, a large study of the United States general population (2001-2002) found 12-month prevalences of $2.6 \%, 2.3 \%$, and $4.9 \%$ for alcohol abuse, dependence, and AUDs, respectively, among females. Among males, these prevalences were $6.9 \%, 5.4 \%$, and $12.3 \%$, respectively. ${ }^{11}$

The discrepancy in the estimates between the populationbased samples and primary care recipients deserves exploration. Using survey data from the United States general population, Cherpitel and Ye estimated the probability of primary care attendance by alcohol users, using a gradient grouping for alcohol use (nonrisky, risky, problem drinking, and alcohol dependence). Only DSM-IV alcohol dependence was found to be a significant predictor of primary care use (odds ratio $=1.63) .{ }^{4}$ The investigators suggested that alcohol dependence is likely to result in health problems requiring primary care treatment, thus leading to the higher prevalence of alcohol dependence in primary care compared with the general population. Additionally, $3.2 \%$ and $6.6 \%$ of primary care and ED users, respectively, used illicit drugs more than monthly. ${ }^{4}$

\section{Screening \\ What is screening?}

Screening refers to a tool - usually a brief questionnaire - that finds subjects who have or are at high risk for a disorder in a population of interest (eg, primary care). Screening does not establish a diagnosis. Instead, it identifies people at risk for or likely to have a disorder. Effective screening instruments exist for alcohol-related problems in primary care, including CAGE and AUDIT, described below. ${ }^{5,12}$ Screening for alcohol-related problems and disorders, however, is a challenge. This is due in part to the limited time available to primary care physicians, especially in managed-care settings, and the pressure to screen for multiple medical and psychiatric disorders. Concerns about limited time and lack of financing to screen for alcohol and illicit substances or the nonmedical use of prescription drugs, and to intervene with those individuals screening positive, may be relieved by the Patient Protection and Affordable Care Act (PL 111-148, 23 March 2010). For new private health plans and insurance policies, the Act requires coverage of various preventive health interventions, including alcohol and drug-related counseling. ${ }^{13}$

Screening is not applicable to all disorders. Disorders with the following characteristics are suitable for screening: 
(1) the disorder should lead to substantial morbidity, (2) effective treatment should be available, (3) treatment initiated in at-risk individuals or in early stages of the disease should be more effective than later in the disease course, and (4) the disorder should be relatively common because it is not cost-effective to screen for disorders with very low prevalence in the population of interest. ${ }^{12} \mathrm{~A}$ good screening instrument has high "sensitivity" (ie, identifies most cases with the disorder) and high "specificity" (ie, avoids positive screens of individuals without the disorder). Cutoff scores for screening tests are determined so as to maximize sensitivity and specificity, thus minimizing false positives and false negatives. ${ }^{12}$

Screening for substance use disorders (SUDs) and their antecedents can be done using instruments that screen for mental disorders in general and include a substance abuse component, and also with instruments that specifically target alcohol and/or drug misuse. The former category includes numerous instruments such as the PRIME-MD. ${ }^{14}$ To be of practical use in primary care settings, a screening instrument must be brief and easy to score. A few of the most widely used screens that specifically target AUDs and SUDs are briefly summarized here. These instruments have been investigated using longitudinal designs to estimate their predictive ability. Additional alcohol screening tools have been reviewed elsewhere. ${ }^{12}$ Screening instruments for illicit drugs have not been studied in as much depth as yet, but some data are available.

\section{Screening instruments for alcohol misuse and AUDs}

Current practices generally rely on a two-step strategy. ${ }^{15}$ Individuals are assessed initially by a single-item or a twoitem screener for either alcohol or drug use status; the subset of users is then screened for substance use-related problems or disorders.

\section{Screeners for alcohol use and misuse}

The term "misuse" lacks a uniform definition. Therefore, it is defined below as used by the authors of the instruments reviewed here. The term "disorder" is used in this review to refer to substance-related disorders listed in the DSM-IV.

A single-item screener ("How many times in the past year have you had X or more drinks in a day?"; X $=5$ for men and 4 for women) recommended by the National Institute on Alcohol Abuse and Alcoholism has been studied in primary care settings. This question was sensitive $(87.9 \%)$ but less specific (66.8\%) for the detection of a current DSM-IV AUD. ${ }^{16}$ Another initial two-item screener goes beyond alcohol use by focusing on (1) recurrent drinking in hazardous situa- tions and (2) drinking more than intended. If either question is answered affirmatively, the sensitivity for a current SUD varies from $77 \%$ to $95 \%$ and the specificity from $62 \%$ to $86 \%$, depending on the sample used to study this approach. ${ }^{17}$ Commonly used screeners are reviewed here.

\section{CAGE}

CAGE is an acronym referring to the most popular screening instrument for alcohol-related problems, and it has been used extensively in primary care. It consists of four straightforward questions: ${ }^{18}$ (1) "Have you ever felt you ought to Cut down on your drinking?"; (2) "Have people Annoyed you by criticizing your drinking?"; (3) "Have you ever felt bad or Guilty about your drinking?"; and (4) "Have you ever had a drink in the morning to steady your nerves or get rid of a hangover (Eye opener)?". One positive response should raise the possibility of alcohol-related problems, ${ }^{18}$ but most researchers have used two or more positive responses as a screening criterion. ${ }^{19}$ One drawback of the CAGE is that the "cut down," "guilty," and to some extent, "annoyed" items may elicit false-positive responses, especially among those who drank at an early age and no longer drink. To remediate this limitation of the CAGE, investigators often ask whether the problems reported were experienced in the last year. ${ }^{9}$

To assess the usefulness of screeners, investigators often examine their reliability and validity. "Reliability" refers to the consistency of scores and is often measured by having two raters (inter-rater reliability) or by administering a screener twice to the same subject (test-retest reliability). Correlation coefficients $>0.7$ or 0.8 are usually considered adequate. ${ }^{20}$ "Test-retest reliability" is generally high for the CAGE. For example, it was 0.95 and 0.80 in a community and a psychiatric sample, respectively, both with AUDs. ${ }^{21}$ "Validity" refers to the ability of a screener to measure what it purports to measure. Validity is often estimated by comparing results of a screener to diagnoses or assessments obtained by doing a thorough, often standardized, interview and assessing the sensitivity and specificity of a screener. The positive predictive value (PPV) answers a clinically important question - ie, what proportion of those screening positive for a disorder (or problem) have the disorder? A few studies have estimated the sensitivity and specificity of the CAGE. ${ }^{22}$ Unless otherwise noted, this paper reports on studies using a cutoff score $\geq 2$ (two or more questions answered affirmatively). The CAGE does well in primary care settings ${ }^{23}$ but not as well with certain populations (eg, heavy drinkers and college students). ${ }^{20,22}$ Ten primary care studies assessed the sensitivity of and specificity of the CAGE for 
alcohol abuse and/or dependence and found sensitivities of $21 \%-94 \%$ and specificities of $77 \%-97 \% .^{22} \mathrm{~A}$ pooled analysis of the CAGE in screening for alcohol abuse and dependence found a sensitivity of 0.87 in inpatients and of 0.71 in primary care patients. ${ }^{23}$ The CAGE, as is the case with most screeners, yields different results across populations and across settings. For example, a sensitivity of $49 \%-69 \%$ and a specificity of $75 \%-95 \%$ have been reported among heavy drinkers. ${ }^{24,25}$ Recently, the CAGE has been combined with biochemical measures to further enhance its validity. ${ }^{26}$ In one review, the PPV varied from $0.36-0.98$ when considering cutoff scores of $\geq 1$ and $\geq 2$, with the lowest PPV found among college students. ${ }^{20}$ The authors suggested that binge drinking, common among college students, is often not captured by the CAGE.

The specificity and sensitivity of a screener with a binary outcome (eg, diseased versus not diseased) are often plotted in a single graph, known as receiving operating characteristics (ROC) curve. The aim of plotting the ROC is to find a combination of sensitivity and specificity that optimizes the use of a test. Each point on the ROC curve represents a sensitivity/specificity pair. The area under the ROC curve is a measure of how well a screener can distinguish between two diagnostic groups (diseased/normal), with the area under the curve (AUC) reflecting the ability to distinguish cases from noncases using a specific test and a given cutoff. A greater AUC reflects a better ability to make this distinction accurately. The CAGE, when used to screen for alcohol abuse/dependence, has an AUC between 0.75 and 0.94 according to an often-quoted meta-analysis. ${ }^{23}$

\section{The Alcohol Use Disorders Identification Test (AUDIT)}

AUDIT is the most studied screening tool for detecting alcohol-related problems in primary care settings, and the four-item CAGE is among the most popular screening instrument in these settings. ${ }^{7}$ The AUDIT consists of 10 questions that address the extent of alcohol use, its negative consequences, and evidence that others have noticed that the respondent has a drinking problem. ${ }^{27}$ A World Health Organization study found that, among those engaging in hazardous alcohol use, 92\% had an AUDIT score of 8 or more, and $94 \%$ of those with nonhazardous consumption had a score of less than $8,{ }^{27}$ suggesting that the AUDIT is a valid instrument with high sensitivity and specificity.

A shorter version, known as AUDIT-C, has also been used in primary care. It consists of items $1-3$ of the 10 -item AUDIT. These items focus on alcohol consumption and include the following questions: (1) "How often do you have a drink containing alcohol?"; (2) "How many drinks containing alcohol do you have on a typical day when you are drinking?"; and (3) "How often do you have six or more drinks on one occasion?". The answers are assigned points that reflect severity as described elsewhere. ${ }^{5}$

Test-retest reliability studies are difficult to compare because different intervals have been applied in studies of the AUDIT, with some asking about the last month and others about longer intervals. ${ }^{28}$ In a study that focused on the last month using a general population sample, the test-retest reliability was $0.84 .^{29}$ A review of ROC studies of the AUDIT and the AUDIT-C reported an AUC $\geq 0.80$ in most studies reviewed. ${ }^{28}$ A study of American veterans attending general medicine clinics compared the AUDIT and the AUDIT-C. The AUC was greater for the AUDIT (compared with the AUDIT-C) when used to detect active alcohol abuse/dependence ( 0.811 versus 0.786; $P<0.001$ ), suggesting a better performance for the full AUDIT. However, the two questionnaires performed similarly when they were used to detect heavy drinking. ${ }^{30}$

\section{Use of alcohol screeners in primary care}

Screening for any disorder is time-consuming and costly. To justify these efforts, screening tools must be as sensitive and specific as possible, and an intervention must be available for those screening positive for the disorder. Using the CAGE, Chan et al found a sensitivity/specificity of $94.4 \% / 97.0 \%$ for past-year alcohol-related problems in a primary-care setting. ${ }^{9}$ Using a single question, as recommended by the National Institute on Alcohol Abuse and Alcoholism, has good sensitivity $(87.9 \%)$ but limited specificity $(66.8 \%$ for the detection of a current AUD). Nevertheless, the single-question approach may be useful for detecting at-risk individuals below the threshold for a disorder. ${ }^{16}$ A large primary-care sample was studied using the AUDIT-C and a structured interview. The prevalence of risky drinking (as defined by Bradley and described above) was 19\% and 33\% for women and men, respectively; $9 \%$ and 17\% for AUDs. With misuse as the gold standard (defined as risky drinking and/or AUDs), a score of $\geq 3$ and $\geq 4$ in women and men, respectively, was associated with sensitivities/specificities of $0.73 / 0.91$ and $0.86 / 0.89$, respectively, and sensitivity/specificity values were higher for AUDs compared with misuse. ${ }^{5}$

\section{Screening instruments for drug use and drug use disorders Screening instruments for drug use}

A single-item drug use screener was recently reported. ${ }^{31}$ The question ("How many times in the past year have you 
used an illegal drug or used a prescription medication for nonmedical reasons?") was highly sensitive (100\%; 95\% confidence interval [CI] 90.6\%-100\%) but not highly specific $(73.5 \%$; 95\% CI $67.7 \%-78.6 \%)$ for the detection of a drug use disorder among adults recruited from primary care waiting rooms. The TICS (Two-Item Conjoint Screen) is a two-item screener targeting alcohol and drug use. ${ }^{32}$ The two questions are: "In the last year, have you ever drunk or used drugs more than you meant to?" and "Have you felt you wanted or needed to cut down on your drinking or drug use in the last year?" At least one positive response detected current substance use disorders with nearly $80 \%$ sensitivity and specificity. ${ }^{32}$

\section{Screening for drug use disorders}

This section focuses on the most commonly used instrument to screen for drug use problems or disorders - the DAST (Drug Abuse Screening Test). The original version of this self-report includes 28 items that tap consequences or problems related to drug use and are combined to obtain a total DAST score. This score represents a quantitative estimate of problems associated with drug use. ${ }^{33}$ The DAST has good concurrent and discriminant validity, as well as good reliability and validity, ${ }^{34,35}$ and has been used extensively in primary-care settings. Using a cutoff score of 6 as indicative of drug misuse, as suggested in the original publication, ${ }^{33}$ studies have found sensitivities of $80.9 \%-96 \%$ and specificities of $71 \%-93.9 \%{ }^{35}$ In addition to the original 28 -item version, shorter versions including 10 and 20 items are available. ${ }^{35}$

\section{Brief interventions in primary care settings}

The USPSTF has recommended screening and behavioral counseling in primary care to reduce unhealthy alcohol use by adults. ${ }^{7}$ Most randomized trials of brief interventions published before 2008 are cited in a review of their cost-effectiveness, ${ }^{36}$ and a few more recent studies are discussed below.

Many of these brief interventions have common components, including the use of educational materials and information, motivational interviewing (MI), contracting, and diaries, and they may include take-home exercises. MI was designed as a way to help people work through ambivalence and commit to change in a supportive environment. ${ }^{37} \mathrm{MI}$ counseling brings to the forefront the ambivalence of patients by discussing the motivation not to change, as well as the motivation to change. In the case of excessive alcohol use, a counselor might ask, for example, about what the patient enjoys about drinking large amounts of alcohol and what bothers him about drinking too much, with the goal of facilitating the patient's commitment to change. Contracting refers to patients' agreements to commit to a specific change (eg, to cut down from six to three drinks per occasion). Diaries are encouraged because they offer a concrete way of documenting change, while at the same time enhancing motivation for change. Take-home exercises vary widely but often include readings and assignments. Patients may be asked to complete worksheets and bring them back for discussion. ${ }^{38}$

A few studies are briefly discussed among the many focusing on brief interventions to reduce excessive alcohol use in primary care. First, a study of primary-care patients in Wisconsin recruited adults aged 18-65 years coming for routine care.$^{39}$ Those who screened positive for risky alcohol use, and were otherwise eligible and consenting, participated in a study of a brief intervention to reduce alcohol consumption. The intervention consisted of two 15-minute sessions with their physicians scheduled 1 month apart, and two 5-minute follow-up calls from a nurse after each physician-intervention session. Patients in the active condition experienced a significantly greater reduction in alcohol use and binge drinking compared with the control group, and the intervention effect was maintained over the 48-month follow-up period. The physicians engaged in psycho-education - including themes such as the prevalence of problem drinking and the adverse effects of alcohol - and gave homework as well as a drinking agreement in the form of a prescription.

Second, in a study of United States and Canadian college students who drank excessively, the intervention consisted of two 15-minute counseling sessions, and two follow-up phone calls. This intervention used MI, contracting, diary cards, and take-home exercises. ${ }^{40}$ The intervention led to a moderate decrease of drinking at the 12-month follow-up. A third and last example of studies of brief interventions to reduce excessive alcohol use recruited Australian primarycare patients $(\mathrm{N}=378$ excessive drinkers). The study tested a structured behavioral intervention known as "alcohol screen. ${ }^{\prime 11}$ The intervention included a recommended reading, a diary for monitoring alcohol consumption for a week (to be discussed at the second visit of the active intervention), and three follow-up visits over a 5-month interval. Counseling techniques included MI, psycho-education, instructions about coping with high-risk situations, a discussion of alternative activities associated with a healthier lifestyle, and other advice on relapse prevention. An intention-to-treat analysis that included all patients randomized to the intervention did 
not show a greater reduction of alcohol use in the active compared with the control conditions (minimal intervention and nonintervention). However, among patients returning for a second visit, greater reductions in drinking were evident in the active condition.

In contrast to the many alcohol-related brief interventions for use in primary care, until recently there were very few studies of brief drug abuse interventions in these settings. A large (459,599 patients screened) study of SBIRT compared illicit drug and alcohol use at baseline and 6 months after SBIRT in medical settings in six US states. ${ }^{42}$ The intervention resulted in significant reductions in substance use. Among those using illicit drugs at baseline, rates of drug use at 6 months were $67.7 \%$ lower $(P<0.001)$, and heavy alcohol use was $38.6 \%$ lower $(P<0.001)$. These reductions in self-reported substance use were evident across a range of health care settings and patients. ${ }^{42}$

Although considerable progress has been made in the study of SBIRT to reduce substance abuse, research gaps remain. Most importantly, there are few trials of SBIRT applied to drug use and abuse in primary care. ${ }^{43}$ Because drug use is common, and its negative health impact is known, studies of SBIRT in primary care are needed. ${ }^{44}$

\section{EDs}

\section{Prevalence of substance use problems and disorders in EDs}

Recent data from national surveys of American adults have demonstrated that ED users had higher 1-year prevalences than ED nonusers of coexisting alcohol-drug use (15.2\% versus $12.1 \%$ ), drug use (any drug, 16.9\% versus $13.0 \%$; marijuana, $12.1 \%$ versus $9.7 \%$; prescription opioids, $6.6 \%$ versus $4.1 \%$ ), and alcohol or drug disorders (11.0\% versus 8.5\%). ${ }^{45}$ Among substance users, ED users had higher conditional rates of SUDs than ED nonusers: alcohol or drug disorders among alcohol or drug users, $15.9 \%$ versus $11.7 \%$; marijuana disorders among marijuana users, $16.6 \%$ versus $13.2 \%$; cocaine disorders among cocaine users, $33.2 \%$ versus $22.3 \%$; prescription opioid disorders among nonmedical prescription opioid users, $20.6 \%$ versus $10.0 \%$; stimulant disorders among nonmedical stimulant users, $18.6 \%$ versus 9.2\%; sedative disorders among nonmedical sedative users, $35.0 \%$ versus $4.4 \%$; tranquilizer disorders among nonmedical tranquilizer users, $12.4 \%$ versus $5.2 \% .{ }^{45}$ However, substance use problems among ED attendees may go unrecognized because many patients do not reveal their use of substances, a phenomenon known as "undeclared substance use" or simply "denial."46-48 Thus, prevalence estimates based on recorded substance abuse diagnoses are likely to underestimate the prevalence of use and abuse, as demonstrated using toxicological screening. ${ }^{48}$

In 2008, over 118 million ED visits were made in the United States. Of these, over 4.3 million (about 3.6\%) were associated with drug use. Both the nonmedical use of pharmaceuticals and the use of illicit drugs were involved with ED visits, with cocaine being the most prevalent among illicit drugs (48.5\%). In addition to the direct effects of substances, there were drug-related accidents, suicide attempts, and individuals seeking detoxification. Most of these visits were made by males (62.9\% in 2008), and pharmaceuticals were involved in about two-thirds of ED visits among those aged 20 years and younger and those 65 years or older. ${ }^{1,49,50}$ These trends continued in $2009 .{ }^{51}$ Recently, an alarming increase in deaths due to overdoses of prescription drugs (mainly opioid analgesics) has been reported, and the drugs most often implicated were oxycodone, hydrocodone, and methadone. ${ }^{52}$ Efforts to manage pain more aggressively using opioid analgesics may have inadvertently contributed to increases in opioid-related adverse consequences. ${ }^{52,53}$

\section{SBIRT in EDs}

The ED is the point of entry into the health care system for a variety of patients, including those who drink excessively. ${ }^{54}$ For some patients, the ED may be their only contact with treatment providers. Therefore, the importance of intervening in the ED was recognized during the 2009 Academic Emergency Medicine consensus conference. ${ }^{54}$

The effectiveness of intervention in the ED has been mixed. ${ }^{54}$ Some interventions have been effective at least for a short time, ${ }^{55}$ and others have not. ${ }^{56}$ Before the development of brief interventions, programs often referred patients to treatment. Because referrals were often ignored, interventions to ensure that patients receive substance abuse treatment have been developed. The ED visit is seen as an opportunity for intervention and treatment referral. During these visits, patients are screened, and a brief intervention is delivered to those screening positive for substance abuse. Typically, interventions consist of goal-setting within safe limits, discharge instructions, and a referral to primary care. For those who are substance-dependent or probably dependent, a referral for further assessment is provided and is often followed by substance abuse treatment. There are several SBIRT ED protocols. For example, using health promotion advocates, a program known as project Alcohol and Substance Abuse 
Services Education and Referral to Treatment (ASSERT) successfully negotiated the referral of patients to substance abuse treatment programs. ${ }^{57}$ Successful referrals (ie, referrals that lead to treatment enrolment) are seldom achieved by giving patients a list of treatment resources. Instead, a more active approach is needed. ${ }^{58}$ In project ASSERT, $65 \%$ of those referred to a substance abuse treatment facility through an active negotiation process had enrolled in treatment a month after the referral. Enrolment was about 30 times more likely among ASSERT participants than among those who were indirectly referred for treatment. ${ }^{57,58}$

Alcohol misuse has often been defined in British studies as testing positive for the FAST (Fast Alcohol Screening Test) or the PAT (Paddington Alcohol Test). ${ }^{59,60}$ The FAST ${ }^{59}$ includes the following four questions from the AUDIT: (1) "How often do you have six or more drinks on one occasion?"; (2) "How often during the last year have you been unable to remember what happened the night before because you had been drinking?"; (3) "How often during the last year have you failed to do what was normally expected of you because of drinking"; and (4) "Has a relative or friend, or a doctor or other health worker been concerned about your drinking or suggested you cut down?" Using the AUDIT as the gold standard, the FAST was estimated to have $92.8 \%$ sensitivity and $87.6 \%$ specificity. ${ }^{59}$

The PAT first asks ED patients if they drink alcohol. Those responding affirmatively are asked the following three follow-up questions: ${ }^{60}$

1. "Quite a number of people have times when they drink more than usual; What is the most you will drink in any one day?" The interviewer checks the type and amount of alcohol consumed and estimates the total number of British units of alcohol;

2. If this amount exceeds eight units per day, the interviewer asks "Does this happen once a week or more?" If yes, "Does this happen every day?" If no: "Does this happen at least once a month?"; and

3. "Do you feel your current attendance is related to alcohol?"

Drinking more than eight and six British units of alcohol at least once a week for men and women, respectively, in a single occasion, or stating that the visit to the ED could be related to alcohol are considered indicative of alcohol misuse. Crawford et al completed a study of SBIRT in the United Kingdom targeting PAT-positive ED attendees. Their brief intervention consisted of referring these individuals for an appointment with an alcohol health worker, and they have shown that this intervention results in statistically significant decreases in alcohol consumption evident at 6 but not 12 months post-intervention. ${ }^{61}$

Recent efforts to advance research in this area include the development of a protocol to study SBIRT in the ED context as part of the National Institute on Drug Abuse's National Drug Abuse Treatment Clinical Trials Network. ${ }^{62}$ The many challenges to these studies include defining the study population, the screenings, the interventions to be studied, and the outcomes to be used to estimate their efficacy.

\section{Recent developments The Patient Protection and Affordable Care Act}

The Patient Protection and Affordable Care Act supports preventive services and integration of substance abuse interventions and treatments into the mainstream health care system. Thus, effective screening and intervention for substance use disorders in health care settings are now a priority. The use of biomarkers and of advanced technologies has been recently applied to screening, albeit mostly as investigational tools. These tools may be used in clinical practice in coming years. They are summarized here.

\section{Biomarkers}

A compound known as carbohydrate-deficient transferrin (CDT) is the most well known among those being studied as a biomarker for excessive alcohol use. ${ }^{63,64}$ Although liver enzymes are often elevated in individuals who drink excessively, their usefulness as a screening tool is limited by their lack of ethanol specificity. Other causes of elevated liver enzymes are relatively common (eg, being overweight is often associated with abnormal liver enzyme levels).$^{65}$ Although objective, the use of biomarkers is not without limitations. Urine analysis, the currently preferred drugscreening method, is limited (with few exceptions) to the detection of drug use within a few days before the test, and as in most tests, there are false positives and false negatives, as well as technical problems (eg, the appropriate handling of body fluids).$^{66}$

\section{Advanced technologies}

There is some evidence that web-based screening and brief interventions to limit drinking are effective. These studies have been done in settings where patients are familiar with computer-based applications (eg, a university health care service). ${ }^{67}$ Whether they are applicable to settings with low computer literacy is debatable. Another technology that has been used in this context is interactive voice response 
(IVR) technology ${ }^{68}$ This technology automates the screening and brief intervention and seems to have an impact on reducing drinking, although little is known about the sustainability of IVR-based interventions.

\section{Conclusions and recommendations}

The extant literature supports the usefulness of screening for AUDs and their antecedents in primary care. Two tests, AUDIT and CAGE, generally perform better than other methods (eg, asking about the frequency and quantity of alcohol use). ${ }^{22}$ The role of biomarkers and of advanced technologies in screening deserves further study before these newer approaches are deemed effective. Screening should be used only in settings where an intervention can be delivered immediately or shortly after screening is done. Brief interventions have been shown to be effective to cut drinking among primary care patients with risky or excessive alcohol use. The exception is those who are already alcoholdependent. These individuals need specialized interventions to deal with their disorder. ${ }^{69}$ As mentioned above, there is emerging evidence that SBIRT targeting drug use may also be effective in primary care.

Lack of funding has been an impediment to screening for substance abuse. Funding is likely to become available in the near future, when the Patient Protection and Affordable Care Act is fully implemented. Having enough treatment facilities for those screening positive and referred for treatment will be a challenge once screening is widely implemented. Directions for future research include the study of screening instruments for illicit drug use to achieve a knowledge base comparable to what is known about screening for alcoholrelated problems. ${ }^{70}$ Once screening instruments are deemed effective, the study of brief interventions to deal with illicit drug use in primary care and medical settings should follow. Last, continuous research to identify biomarkers that are easy to use, noninvasive, reliable, and cost-effective is important for their potential use as screening instruments and, perhaps more importantly, as treatment aids. The civil rights and legal implications of using biomarkers as screening instruments merits the attention of ethicists and legal experts.

\section{Acknowledgments}

This paper was made possible by research grants from the US National Institute on Drug Abuse of the National Institutes of Health (R33DA027503, R01DA019623, R01DA019901, and R01DA026652). The National Institutes of Health had no role in the preparation, review, or approval of the manuscript. Its contents are solely the responsibility of the authors and do not necessarily represent the official views of the National Institutes of Health. The authors thank Amanda McMillan, for her editorial assistance.

\section{Disclosure}

The authors have no conflicts of interest to disclose.

\section{References}

1. Substance Abuse and Mental Health Services Administration. Results from the 2010 National Survey on Drug Use and Health: summary of national findings. NSDUH Series H-41, HHS Publication No. (SMA) 11-4658. Rockville, MD: Substance Abuse and Mental Health Services Administration; 2011.

2. Reid MC, Fiellin DA, O'Connor PG. Hazardous and harmful alcohol consumption in primary care. Arch Intern Med. 1999;159:1681-1689.

3. Brick J. Handbook of the medical consequences of alcohol and drug abuse. 2nd ed. New York, NY: The Haworth Press/Taylor and Francis Group; 2008:638.

4. Cherpitel CJ, Ye Y. Drug use and problem drinking associated with primary care and emergency room utilization in the US general population: data from the 2005 national alcohol survey. Drug Alcohol Depend. 2008;97:226-230.

5. Bradley KA, DeBenedetti AF, Volk RJ, Williams EC, Frank D, Kivlahan DR. AUDIT-C as a brief screen for alcohol misuse in primary care. Alcohol Clin Exp Res. 2007;31:1208-1217.

6. D'Amico EJ, Paddock SM, Burnam A, Kung FY. Identification of and guidance for problem drinking by general medical providers: results from a national survey. Med Care. 2005;43:229-236.

7. US Preventive Services Task Force. Screening and behavioral counseling interventions in primary care to reduce alcohol misuse: recommendation statement. Ann Intern Med. 2004;140:554-556.

8. Cunningham RM, Harrison SR, McKay MP, et al. National survey of emergency department alcohol screening and intervention practices. Ann Emerg Med. 2010;55:556-562.

9. Chan AW, Pristach EA, Welte JW. Detection by the CAGE of alcoholism or heavy drinking in primary care outpatients and the general population. $J$ Subst Abuse. 1994;6:123-135.

10. Manwell LB, Fleming MF, Johnson K, Barry KL. Tobacco, alcohol, and drug use in a primary care sample: 90 -day prevalence and associated factors. J Addict Dis. 1998;17:67-81.

11. Grant BF, Dawson DA, Stinson FS, Chou SP, Dufour MC, Pickering RP. The 12-month prevalence and trends in DSM-IV alcohol abuse and dependence: United States, 1991-1992 and 2001-2002. Drug Alcohol Depend. 2004;74:223-234.

12. Stewart SH, Connors GJ. Screening for alcohol problems: what makes a test effective? Alcohol Res Health. 2004;28:5-16.

13. Koh HK, Sebelius KG. Promoting prevention through the Affordable Care Act. N Engl J Med. 2010;363:1296-1299.

14. Spitzer RL, Williams JB, Kroenke K, Hornyak R, McMurray J. Validity and utility of the PRIME-MD patient health questionnaire in assessment of 3000 obstetric-gynecologic patients: the PRIME-MD Patient Health Questionnaire Obstetrics-Gynecology Study. Am J Obstet Gynecol. 2000;183:759-769.

15. Whitlock EP, Green CA, Polen MR, et al. Behavioral counseling interventions in primary care to reduce risky/harmful alcohol use. Rockville, MD: Agency for Healthcare Research and Quality; 2004.

16. Smith PC, Schmidt SM, Allensworth-Davies D, Saitz R. Primary care validation of a single-question alcohol screening test. J Gen Intern Med. 2009;24:783-788.

17. Vinson DC, Kruse RL, Seale JP. Simplifying alcohol assessment: two questions to identify alcohol use disorders. Alcohol Clin Exp Res. 2007;31:1392-1398.

18. Ewing JA. Detecting alcoholism. The CAGE questionnaire. JAMA. 1984;252:1905-1907. 
19. Mayfield D, McLeod G, Hall P. The CAGE questionnaire: validation of a new alcoholism screening instrument. Am J Psychiatry. 1974;131:1121-1123.

20. Dhalla S, Kopec JA. The CAGE questionnaire for alcohol misuse: a review of reliability and validity studies. Clin Invest Med 2007;30:33-41.

21. Teitelbaum LM, Carey KB. Temporal stability of alcohol screening measures in a psychiatric setting. Psychol Addict Behav. 2000;14:401-404.

22. Fiellin DA, Reid MC, O'Connor PG. Screening for alcohol problems in primary care: a systematic review. Arch Intern Med. 2000;160:1977-1989.

23. Aertgeerts B, Buntinx F, Kester A. The value of the CAGE in screening for alcohol abuse and alcohol dependence in general clinical populations: a diagnostic meta-analysis. J Clin Epidemiol. 2004;57:30-39.

24. Aithal GP, Thornes H, Dwarakanath AD, Tanner AR. Measurement of carbohydrate-deficient transferrin (CDT) in a general medical clinic: is this test useful in assessing alcohol consumption. Alcohol Alcohol. 1998;33:304-309.

25. Bradley KA, Bush KR, McDonell MB, Malone T, Fihn SD. Screening for problem drinking: comparison of CAGE and AUDIT. Ambulatory Care Quality Improvement Project (ACQUIP). Alcohol Use Disorders Identification Test. J Gen Intern Med. 1998;13:379-388.

26. do Amaral RA, Malbergier A. Effectiveness of the CAGE questionnaire, gamma-glutamyltransferase and mean corpuscular volume of red blood cells as markers for alcohol-related problems in the workplace. Addict Behav. 2008;33:772-781.

27. Saunders JB, Aasland OG, Babor TF, de la Fuente JR, Grant M. Development of the Alcohol Use Disorders Identification Test (AUDIT): WHO Collaborative Project on Early Detection of Persons with Harmful Alcohol Consumption-II. Addiction. 1993;88:791-804.

28. de Meneses-Gaya C, Zuardi AW, Loureiro SR, Crippa JA. Alcohol Use Disorders Identification Test (AUDIT): an updated systematic review of psychometric properties. Psychol Neurosci. 2009;2:83-97.

29. Selin KH. Test-retest reliability of the alcohol use disorder identification test in a general population sample. Alcohol Clin Exp Res. 2003;27:1428-1435.

30. Bush K, Kivlahan DR, McDonell MB, Fihn SD, Bradley KA The AUDIT alcohol consumption questions (AUDIT-C): an effective brief screening test for problem drinking. Ambulatory Care Quality Improvement Project (ACQUIP). Alcohol Use Disorders Identification Test. Arch Intern Med. 1998;158:1789-1795.

31. Smith PC, Schmidt SM, Allensworth-Davies D, Saitz R. A singlequestion screening test for drug use in primary care. Arch Intern Med. 2010;170:1155-1160.

32. Brown RL, Leonard T, Saunders LA, Papasouliotis O. A two-item conjoint screen for alcohol and other drug problems. J Am Board Fam Pract. 2001;14:95-106.

33. Skinner HA. The drug abuse screening test. Addict Behav. 1982;7:363-371

34. Gavin DR, Ross HE, Skinner HA. Diagnostic validity of the drug abuse screening test in the assessment of DSM-III drug disorders. Br JAddict. 1989;84:301-307.

35. Yudko E, Lozhkina O, Fouts A. A comprehensive review of the psychometric properties of the Drug Abuse Screening Test. J Subst Abuse Treat. 2007;32:189-198.

36. Solberg LI, Maciosek MV, Edwards NM. Primary care intervention to reduce alcohol misuse ranking its health impact and cost effectiveness. Am J Prev Med. 2008;34:143-152.

37. Hettema J, Steele J, Miller WR. Motivational interviewing. Annu Rev Clin Psychol. 2005;1:91-111.

38. Grossberg P, Halperin A, Mackenzie S, Gisslow M, Brown D, Fleming M. Inside the physician's black bag: critical ingredients of brief alcohol interventions. Subst Abus. 2010;31:240-250.

39. Fleming MF, Mundt MP, French MT, Manwell LB, Stauffacher EA, Barry KL. Brief physician advice for problem drinkers: long-term efficacy and benefit-cost analysis. Alcohol Clin Exp Res. 2002;26:36-43.
40. Fleming MF, Balousek SL, Grossberg PM, et al. Brief physician advice for heavy drinking college students: a randomized controlled trial in college health clinics. J Stud Alcohol Drugs. 2010;71:23-31.

41. Richmond R, Heather N, Wodak A, Kehoe L, Webster I. Controlled evaluation of a general practice-based brief intervention for excessive drinking. Addiction. 1995;90:119-132.

42. Madras BK, Compton WM, Avula D, Stegbauer T, Stein JB, Clark H. Screening, brief interventions, referral to treatment (SBIRT) for illicit drug and alcohol use at multiple healthcare sites: comparison at intake and 6 months later. Drug Alcohol Depend. 2009;99:280-295.

43. Saitz R. Alcohol screening and brief intervention in primary care: absence of evidence for efficacy in people with dependence or very heavy drinking. Drug Alcohol Rev. 2010;29:631-640.

44. Tai B, Wu LT, Clark HW. Electronic health records: essential tools in integrating substance abuse treatment with primary care. Subst Abuse Rehabil. 2012;3:1-8.

45. Wu LT, Swartz MS, Wu Z, Mannelli P, Yang C, Blazer DG. Alcohol and drug use disorders among adults in emergency department settings in the United States. Ann Emerg Med. 2012 Mar 14. [Epub ahead of print] PMID: 22424657.

46. Rockett IR, Putnam SL, Jia H, Smith GS. Declared and undeclared substance use among emergency department patients: a populationbased study. Addiction. 2006;101:706-712.

47. Rockett IR, Putnam SL, Jia H, Chang CF, Smith GS. Unmet substance abuse treatment need, health services utilization, and cost: a population-based emergency department study. Ann Emerg Med. 2005;45:118-127.

48. Rockett IR, Putnam SL, Jia H, Smith GS. Assessing substance abuse treatment need: a statewide hospital emergency department study. Ann Emerg Med. 2003;41:802-813.

49. Substance Abuse and Mental Health Services Administration, Center for Behavioral Health Statistics and Quality. Drug Abuse Warning Network, 2008: national estimates of drug-related emergency department visits. In: HHS Publication No. SMA 11-4618. Rockville, MD; 2011.

50. D'Onofrio G, Becker B, Woolard RH. The impact of alcohol, tobacco, and other drug use and abuse in the emergency department. Emerg Med Clin North Am. 2006;24:925-967.

51. Substance Abuse and Mental Health Services Administration, Center for Behavioral Health Statistics and Quality. The DAWN Report: highlights of the 2009 Drug Abuse Warning Network (DAWN) findings on drug-related emergency department visits. Rockville, MD; 2011.

52. Cai R, Crane E, Poneleit K, Paulozzi L. Emergency department visits involving nonmedical use of selected prescription drugs in the United States, 2004-2008. J Pain Palliat Care Pharmacother. 2010;24:293-297.

53. Paulozzi LJ, Budnitz DS, Xi Y. Increasing deaths from opioid analgesics in the United States. Pharmacoepidemiol Drug Saf. 2006; 15:618-627.

54. Cunningham RM, Bernstein SL, Walton M, et al. Alcohol, tobacco, and other drugs: future directions for screening and intervention in the emergency department. Acad Emerg Med. 2009;16:1078-1088.

55. Academic ED SBIRT Research Collaborative. The impact of screening, brief intervention, and referral for treatment in emergency department: patient's alcohol use at 3, 6, and 12 month follow-up. Alcohol Alcohol. 2010;45:514-519.

56. D’Onofrio G, Pantalon MV, Degutis LC, et al. Brief intervention for hazardous and harmful drinkers in the emergency department. Ann Emerg Med. 2008;51:742-750. e2.

57. D'Onofrio G, Degutis LC. Integrating Project ASSERT: a screening, intervention, and referral to treatment program for unhealthy alcohol and drug use into an urban emergency department. Acad Emerg Med. 2010;17:903-911.

58. Bernstein E, Bernstein J, Levenson S. Project ASSERT: an EDbased intervention to increase access to primary care, preventive services, and the substance abuse treatment system. Ann Emerg Med. 1997;30:181-189.

59. Hodgson RJ, John B, Abbasi T, et al. Fast screening for alcohol misuse. Addictive Behaviors. 2003;28(8):1453-1463. 
60. Patton R, Hilton C, Crawford MJ, Touquet R. The Paddington Alcohol Test: a short report. Alcohol Alcohol. 2004;39(3):266-268.

61. Crawford MJ, Patton R, Touquet R, et al. Screening and referral for brief intervention of alcohol-misusing patients in an emergency department: a pragmatic randomised controlled trial. Lancet. 2004;364(9442):1334-1339.

62. Bogenschutz MP, Donovan DM, Adinoff B, et al. Design of NIDA CTN Protocol 0047: screening, motivational assessment, referral, and treatment in emergency departments (SMART-ED). Am J Drug Alcohol Abuse. 2011;37:417-425.

63. Bianchi V, Ivaldi A, Raspagni A, Arfini C, Vidali M. Use of carbohydrate-deficient transferrin (CDT) and a combination of GGT and CDT (GGT-CDT) to assess heavy alcohol consumption in traffic medicine. Alcohol Alcohol. 2010;45:247-251.

64. Allen JP, Litten RZ. The role of laboratory tests in alcoholism treatment. J Subst Abuse Treat. 2001;20:81-85.

65. Niemela O, Alatalo P. Biomarkers of alcohol consumption and related liver disease. Scand J Clin Lab Invest. 2010;70:305-312.
66. Babor TF, Kadden RM. Screening and interventions for alcohol and drug problems in medical settings: what works? J Trauma. 2005;59:S80-S87; discussion S94-S100.

67. Kypri K, Langley JD, Saunders JB, Cashell-Smith ML, Herbison P. Randomized controlled trial of web-based alcohol screening and brief intervention in primary care. Arch Intern Med. 2008;168:530-536.

68. Rose GL, MacLean CD, Skelly J, Badger GJ, Ferraro TA, Helzer JE. Interactive voice response technology can deliver alcohol screening and brief intervention in primary care. J Gen Intern Med. 2010;25:340-344.

69. Saitz R, Alford DP, Bernstein J, Cheng DM, Samet J, Palfai T. Screening and brief intervention for unhealthy drug use in primary care settings: randomized clinical trials are needed. $J$ Addict Med. 2010;4:123-130.

70. Wu LT, Blazer DG, Woody GE, et al. Alcohol and drug dependence symptom items as brief screeners for substance use disorders: results from the Clinical Trials Network. J Psychiatr Res. 2012;46:360-369.
Substance Abuse and Rehabilitation

\section{Publish your work in this journal}

Substance Abuse and Rehabilitation is an international, peer-reviewed, open access journal publishing original research, case reports, editorials, reviews and commentaries on all areas of addiction and substance abuse and options for treatment and rehabilitation. The manuscript management system is completely online and includes a very quick and fair

\section{Dovepress}

peer-review system. Visit http://www.dovepress.com/testimonials.php to read real quotes from published authors. 